PROCEEDINGS OF THE

AMERICAN MATHEMATICAL SOCIETY

Volume 125, Number 8, August 1997, Pages 2435-2441

S 0002-9939(97)03796-9

\title{
SOUSLIN TREES WHICH ARE HARD TO SPECIALISE
}

\author{
JAMES CUMMINGS \\ (Communicated by Andreas R. Blass)
}

\begin{abstract}
We construct some $\kappa^{+}$-Souslin trees which cannot be specialised by any forcing which preserves cardinals and cofinalities. For $\kappa$ a regular cardinal we use the $\otimes$ principle, and for $\kappa$ singular we use squares and diamonds.
\end{abstract}

\section{INTRODUCTION}

We start by recalling a few basic definitions concerning trees. For more information, see [4].

Definition 1.1. Let $\kappa$ be a regular cardinal.

1. $\left(T,<_{T}\right)$ is a tree iff $<_{T}$ is a partial ordering of $T$ such that $\left\{y: y<_{T} x\right\}$ is well ordered by $<_{T}$ for all $x \in T$.

2. Let $T$ be a tree. Then

(a) If $x \in T, \operatorname{ht}_{T}(x)$ is the order type of $\left(\left\{y: y<_{T} x\right\},<_{T}\right)$.

(b) $T_{\alpha}=\left\{x \in T: \operatorname{ht}_{T}(x)=\alpha\right\}$.

(c) $\operatorname{ht}(T)$ is the least $\alpha$ such that $T_{\alpha}=\emptyset$.

(d) $T \uparrow \alpha=\bigcup_{\beta<\alpha} T_{\beta}$.

(e) A cofinal branch of $T$ is a set $B \subseteq T$ such that $B$ is linearly ordered by $<_{T}$, and $\forall \alpha<\operatorname{ht}(T) \exists b \in B \operatorname{ht}_{T}(b) \geq \alpha$.

3. $T$ is a $\kappa$-tree iff $h t(T)=\kappa$ and $\left|T_{\alpha}\right|<\kappa$ for all $\alpha<\kappa$.

4. $T$ is a $\kappa$-Aronszajn tree iff $T$ is a $\kappa$-tree with no cofinal branch.

5. $T$ is a special $\kappa^{+}$-tree iff $T$ is a $\kappa^{+}$-tree and there exists $F: T \longrightarrow \kappa$ such that $x<_{T} y \Longrightarrow F(x) \neq F(y)$.

6. $X \subseteq T$ is an antichain in $T$ iff for all $x, y \in X$ with $x \neq y$ we have $x \varliminf_{T} y$ and $y \not_{T} x$.

7. $T$ is a $\kappa$-Souslin tree iff $T$ is a $\kappa$-Aronszajn tree and $T$ has no antichain of size $\kappa$.

It is easy to see that a special $\kappa^{+}$-tree is an $\kappa^{+}$-Aronszajn tree and that a $\kappa^{+}$Souslin tree is a non-special $\kappa^{+}$-Aronszajn tree. Sometimes it is possible to make trees special by mild forcing; for example if $T$ is an $\aleph_{1}$-Aronszajn tree then it is proved in [1] that there exists a c.c.c forcing $\mathbb{P}_{T}$ which adds a specialising function for $T$.

Received by the editors September 7, 1995 and, in revised form, February 12, 1996.

1991 Mathematics Subject Classification. Primary 03E05; Secondary 03E35.

Key words and phrases. Souslin trees, ascent paths, squares and diamonds.

The author was supported by a Postdoctoral Fellowship at the Mathematics Institute, Hebrew University of Jerusalem.

(C) 1997 American Mathematical Society 
We will construct some $\lambda^{+}$-Souslin trees which are hard to specialise. The key idea will be that of an ascent path, which is due to Laver.

Definition 1.2. Let $\kappa$ and $\lambda$ be cardinals with $\kappa=\operatorname{cf}(\kappa)<\lambda$. Let $T$ be a $\lambda^{+}$-tree. Then a $\kappa$-ascent path in $T$ is a sequence $\left\langle x_{\alpha}: \alpha \in A\right\rangle$ such that

1. $A$ is unbounded in $\lambda^{+}$.

2. For each $\alpha \in A, x_{\alpha}$ is a function from $\kappa$ to $T_{\alpha}$.

3. If $\alpha, \beta \in A$ with $\alpha<\beta$ then $\exists \gamma<\kappa \forall \delta \geq \gamma x_{\alpha}(\delta)<_{T} x_{\beta}(\delta)$.

The connection between ascent paths and questions about specialisation is given by the following theorem from [6], a paper in which some Aronszajn trees with ascent paths are constructed. The theorem is due to Shelah, building on work of Laver and Todorčević.

Theorem 1.3. Let $T$ be a $\lambda^{+}$-tree with a $\kappa$-ascent path, where $\kappa \neq \operatorname{cf}(\lambda)$. Then $T$ is not special.

Since the hypotheses of this theorem will remain true in any extension of the universe with the same cardinals and cofinalities, it follows that trees with ascent paths are rather hard to specialise.

Baumgartner proved that if $\nabla_{\omega_{2}}(E)$ and $\square_{\omega_{1}}(E)$ hold for some stationary set $E \subseteq \aleph_{2}$, then it is possible to construct an $\aleph_{2}$-Souslin tree with an $\omega$-ascent path. The construction appears in [2], and is a variation on Jensen's construction of an $\aleph_{2}$-Souslin tree from the same hypotheses.

There is another well-known construction of an $\aleph_{2}$-Souslin tree, in which the tree is built using the hypotheses that $\mathrm{CH}$ and $\diamond\left(S_{1}^{2}\right)$ hold (where $S_{1}^{2}$ is the set $\left.\left\{\alpha<\aleph_{2}: \operatorname{cf}(\alpha)=\aleph_{1}\right\}\right)$. In this construction the resulting tree is countably closed, because the stages at which antichains are guessed and dealt with have cofinality $\aleph_{1}$, while all cofinal branches are completed at stages of cofinality $\omega$. In Theorem 2.2 we will show that it is possible to construct a countably closed $\aleph_{2}$-Souslin tree with an $\omega$-ascent path; unfortunately we will need rather stronger assumptions; namely we will need to assume $\mathrm{CH}$ plus the principle $Q$.

Theorem 2.2 has an easy generalisation to successors of regular cardinals, which is given in Theorem 2.4. We will prove in Theorems 3.1 and 3.4 that under the right hypotheses (square and some consequences of GCH) we can build Souslin trees that are hard to specialise at successors of singulars. Here we will use the idea of Baumgartner's construction, plus some results of Shelah concerning diamond principles that follow from the GCH.

\section{SucCESSORS OF REgulars}

In the constructions of this section we will use the combinatorial principle (introduced in [3]).

Definition 2.1. A $\nabla$-sequence is a sequence $\left\langle S_{\alpha}, C_{\alpha}: \alpha<\aleph_{2}\right\rangle$ where

1. $S_{\alpha} \subseteq \alpha$ and $C_{\alpha}$ is club in $\alpha$.

2. ot $\left(C_{\alpha}\right) \leq \aleph_{1}$.

3. $\beta \in \lim \left(C_{\alpha}\right)$ implies $C_{\beta}=C_{\alpha} \cap \beta$ and $S_{\beta}=S_{\alpha} \cap \beta$.

4. If $C$ is club in $\aleph_{2}$ and $X \subseteq \aleph_{2}$ then there exists $\beta$ with $\operatorname{cf}(\beta)=\aleph_{1}$ such that $C_{\beta} \subseteq C$ and $S_{\beta}=X \cap \beta$.

It is shown in [3] that $\otimes$ holds in $L$. can also be forced rather easily; the conditions are just sequences of the form $\left\langle S_{\alpha}, C_{\alpha}: \alpha \leq \beta\right\rangle$ obeying clauses 1-3 of 
the definition, and it is easy to see that the forcing is $\left(\aleph_{1}+1\right)$-strategically closed and that the generic sequence is a 8 -sequence.

Theorem 2.2. Assume that $C H$ and $\otimes$ hold. Then there is a countably closed $\aleph_{2}$-Souslin tree with an $\omega$-ascent path.

Proof. We will build a countably closed $\aleph_{2}$-Souslin tree $T$ together with a function $\alpha \longmapsto x_{\alpha}$ which assigns to each $\alpha<\aleph_{2}$ an $\omega$-sequence $x_{\alpha}$ of points from $T_{\alpha}$. Suppose that $\left\langle S_{\alpha}, C_{\alpha}: \alpha<\aleph_{2}\right\rangle$ is a $\theta$-sequence. We will maintain the following hypotheses during the inductive construction of $T$ and $\vec{x}$.

1. The elements of $T$ are ordinals less than $\aleph_{2}$, and $\left|T_{\alpha}\right| \leq \aleph_{1}$ for all $\alpha$. The ordinals appearing in $T\left\lceil\alpha\right.$ form an initial segment of $\aleph_{2}$.

2. $\alpha<\beta<\aleph_{2} \Longrightarrow \exists n \forall m \geq n x_{\alpha}(m)<_{T} x_{\beta}(m)$.

3. $T \uparrow \alpha$ is countably closed; that is to say, if $x_{0}<_{T} x_{1}<_{T} x_{2} \ldots$ and $\beta=\sup _{n} \operatorname{ht}\left(x_{n}\right)<\alpha$ then there exists $y \in T_{\beta}$ such that $\forall n x_{n}<_{T} y$.

4. $T\lceil\alpha$ is normal; that is,

(a) Every element $x$ with $\operatorname{ht}(x)+1<\alpha$ has two immediate successors in $T_{\mathrm{ht}(x)+1}$.

(b) If $\rho<\sigma<\alpha$ and $x \in T_{\rho}$ then there is $y \in T_{\sigma}$ with $x<y$.

(c) If $\lambda$ is a limit ordinal less than $\alpha$, and $x, y \in T_{\lambda}$ with $x \neq y$, then $\left\{z: z<_{T} x\right\} \neq\left\{z: z<_{T} y\right\}$.

5. $\alpha \in \lim \left(C_{\beta}\right) \Longrightarrow \forall m x_{\alpha}(m)<_{T} x_{\beta}(m)$.

Before we begin the construction, a few words of motivation: Hypotheses 1-3 are completely natural in the light of our final goal, and Hypothesis 4 is a standard one in inductive constructions of trees. Hypothesis 5 is intended to help us maintain Hypothesis 2, which might otherwise be impossible at limit stages of cofinality $\aleph_{1}$. Hypothesis 3 will be maintained by completing all cofinal branches through $T\lceil\alpha$ at every limit stage $\alpha$ of cofinality $\omega$; this will not cause $T_{\alpha}$ to become too large because we are assuming $\mathrm{CH}$.

The main difficulty in the construction is that maintaining Hypothesis 5 will force us to complete certain branches at stages of cofinality $\aleph_{1}$. This will potentially cause conflict with our strategy for making $T$ Souslin, which (as usual) will involve sealing off antichains by not completing certain branches at stages of cofinality $\aleph_{1}$. We will use the powerful guessing properties of the 8 -sequence to resolve the conflict.

We describe the inductive construction of $T$ and $\vec{x}$.

1. $\alpha=0 . T_{0}$ has a single point and $x_{0}$ is constant with value that point.

2. $\alpha=\beta+1$. Put two points of $T_{\alpha}$ over each point of $T_{\beta}$ and for each $n$ designate one of the successors of $x_{\beta}(n)$ to be $x_{\alpha}(n)$.

3. $\operatorname{cf}(\alpha)=\omega$ and $\lim \left(C_{\alpha}\right)$ is unbounded in $\alpha$. Put a point of $T_{\alpha}$ over each cofinal branch of $T\left\lceil\alpha\right.$. If $\gamma, \delta \in \lim \left(C_{\alpha}\right)$ with $\gamma<\delta$ then $\gamma \in \lim \left(C_{\delta}\right)$, so that $x_{\gamma}(n)<_{T} x_{\delta}(n)$. So for each $n$ the sequence $\left\langle x_{\gamma}(n): \gamma \in \lim \left(C_{\alpha}\right)\right\rangle$ is a cofinal branch, and we designate the point of $T_{\alpha}$ on top of that branch as $x_{\alpha}(n)$.

Hypothesis 5 is satisfied by construction. Hypothesis 2 is also satisfied because if $\beta<\alpha$ then for some $\gamma \in \lim \left(C_{\alpha}\right)$ we have $\beta<\gamma<\alpha$; by construction $x_{\beta}(n)<_{T} x_{\gamma}(n)$ for all large $n$ and $x_{\gamma}(n)<_{T} x_{\alpha}(n)$ for all $n$.

4. $\operatorname{cf}(\alpha)=\omega$ and $\lim \left(C_{\alpha}\right)$ is bounded in $\alpha$, say the maximum point is $\gamma$. Notice that for all $\beta \in \lim \left(C_{\alpha}\right)$ and all $n$ we have $x_{\beta}(n) \leq_{T} x_{\gamma}(n)$. Put a point of $T_{\alpha}$ over each cofinal branch of $T\left\lceil\alpha\right.$. Choose an $\omega$-sequence $\left\langle\alpha_{n}: n<\omega\right\rangle$ cofinal in $\alpha$ with $\alpha_{0}=\gamma$. Now for each $n$ let $i(n) \leq n$ be maximal such that 
$x_{\alpha_{0}}(n) \leq_{T} \ldots \leq_{T} x_{\alpha_{i(n)}}(n)$; build a cofinal branch in $T\lceil\alpha$ passing through $x_{\alpha_{i(n)}}(n)$, and such that if possible the branch also passes through a point of $S_{\alpha}$. Designate the point on top of this branch as $x_{\alpha}(n)$.

Hypothesis 5 is satisfied because if $\beta \in \lim \left(C_{\alpha}\right)$ then for all $n$ we have $x_{\beta}(n) \leq_{T} x_{\gamma}(n)<x_{\alpha}(n)$. For Hypothesis 2 let $i<\omega$, and observe that for all large enough $n$ we have $n \geq i$ and $x_{\alpha_{0}}(n)<\ldots<x_{\alpha_{i}}(n)$, so that $i(n) \geq i$ and therefore $x_{\alpha_{i}}(n) \leq_{T} x_{\alpha_{i(n)}}(n)<_{T} x_{\alpha}(n)$. For all $\beta<\alpha$ there is an $i$ such that $\beta<\alpha_{i}<\alpha$, and hence $x_{\beta}(n)<_{T} x_{\alpha_{i}}(n)<_{T} x_{\alpha}(n)$ for all large $n$.

5. $\operatorname{cf}(\alpha)=\aleph_{1}$. For each point $x$ of $T\lceil\alpha$ build a branch which passes through $x$, and also if possible passes through a point of $S_{\alpha}$. Put a point of $T_{\alpha}$ on top of each such branch. For each $m$ the sequence $\left\langle x_{\beta}(m): \beta \in \lim \left(C_{\alpha}\right)\right\rangle$ is a cofinal branch; if we did not do so already, put a point on top of this branch. Now, for each $m$, designate $x_{\alpha}(m)$ as the point over the branch $\left\langle x_{\beta}(m): \beta \in \lim \left(C_{\alpha}\right)\right\rangle$.

As in Case 3, Hypotheses 2 and 5 are easily seen to be satisfied.

It remains to be seen that this works. It is clear that we have satisfied the demands on the $x_{\alpha}$, and built a normal countably closed $\aleph_{2}$-tree, the problem is to see that we have built an $\aleph_{2}$-Souslin tree. By normality it is enough to check that there are no antichains of size $\aleph_{2}$. Let $S$ be a maximal antichain and let $C$ be the club consisting of those $\alpha$ such that $T\lceil\alpha=\alpha$ and $S \cap \alpha$ is maximal in $T\lceil\alpha$. Find $\alpha$ such that $\operatorname{cf}(\alpha)=\aleph_{1}, C_{\alpha} \subseteq C$ and $S \cap \alpha=S_{\alpha}$. Notice that $\alpha=\sup \left(C_{\alpha}\right) \in C$.

Now consider the construction of $T_{\alpha}$; we claim that every point of $T_{\alpha}$ lies over some point of $S_{\alpha}$. We completed branches of $T \uparrow \alpha$ for two reasons, to satisfy normality and to define $x_{\alpha}$. Those branches which we completed to get normality clearly pass through $S_{\alpha}$, because $S_{\alpha}=S \cap \alpha$ is maximal in $T\lceil\alpha$, so for each $x$ it was possible to construct a branch through $x$ and some point of $S_{\alpha}$. To see that the other branches pass through $S$ consider $\beta$ a successor point in $\lim \left(C_{\alpha}\right)$; now $\beta \in C$ so $S_{\beta}=S_{\alpha} \cap \beta=S \cap \beta$ is maximal in $T \uparrow \beta$, and $\lim \left(C_{\beta}\right)=\lim \left(C_{\alpha}\right) \cap \beta$ is bounded in $\beta$, so that when we define $x_{\beta}$ we will have arranged that every point $x_{\beta}(n)$ lies over some point of $S_{\beta}$. It follows that $S_{\alpha}$ is maximal in $T$, and hence that $S=S_{\alpha}$ and $|S| \leq \aleph_{1}$.

This concludes the proof of Theorem 2.2.

The result has an easy generalisation.

Definition 2.3. Let $\kappa$ be a regular cardinal. A $\otimes_{\kappa}$-sequence is a sequence of pairs $\left\langle S_{\alpha}, C_{\alpha}: \alpha<\kappa^{+}\right\rangle$where

1. $S_{\alpha} \subseteq \alpha$ and $C_{\alpha}$ is club in $\alpha$.

2. $\operatorname{ot}\left(C_{\alpha}\right) \leq \kappa$.

3. $\beta \in \lim \left(C_{\alpha}\right)$ implies $C_{\beta}=C_{\alpha} \cap \beta$ and $S_{\beta}=S_{\alpha} \cap \beta$.

4. If $C$ is club in $\kappa^{+}$and $X \subseteq \kappa^{+}$then there exists $\beta$ with $\operatorname{cf}(\beta)=\kappa$ such that $C_{\beta} \subseteq C$ and $S_{\beta}=X \cap \beta$.

Theorem 2.4. Let $\kappa^{<\kappa}=\kappa$ and let $\nabla_{\kappa}$ hold. Then there is a $\kappa^{+}$-Souslin tree with an $\omega$-ascent path.

Proof. Exactly like the proof of Theorem 2.2.

\section{SuCCESSORS OF Singulars}

We now turn to $\lambda^{+}$-trees with $\lambda$ singular. It is hopeless to demand that such a tree be $<\lambda$-closed because $\lambda^{\operatorname{cf}(\lambda)}>\lambda$. We will prove that under the right 
hypotheses there can exist $\lambda^{+}$-Souslin trees with $\kappa$-ascent paths for some $\kappa \neq \operatorname{cf}(\lambda)$. Our construction is very similar to Baumgartner's construction described in [2]. We start by treating the case when $\operatorname{cf}(\lambda)=\omega$.

Theorem 3.1. Let $\lambda$ be a cardinal such that

1. $\operatorname{cf}(\lambda)=\omega$.

2. $\gamma<\lambda \Longrightarrow \gamma^{\aleph_{1}}<\lambda$.

3. $2^{\lambda}=\lambda^{+}$.

4. $\square_{\lambda}$ holds.

Then there exists an $\lambda^{+}$-Souslin tree with an $\aleph_{1}$-ascent path.

Proof. We begin by deriving some consequences of the assumptions. The following is well-known.

Claim 3.2. There exists $E$ a stationary subset of $\left\{\alpha<\lambda^{+}: \operatorname{cf}(\alpha)=\aleph_{1}\right\}$ and a sequence $\left\langle C_{\alpha}: \alpha<\lambda^{+}\right\rangle$such that $\vec{C}$ is a $\square_{\lambda}(E)$-sequence, that is, a $\square_{\lambda}$-sequence with the additional property that $\forall \alpha \lim \left(C_{\alpha}\right) \cap E=\emptyset$.

Proof. Let $\left\langle D_{\alpha}: \alpha\left\langle\lambda^{+}\right\rangle\right.$be a $\square_{\lambda}$-sequence. By Fodor's lemma find $E$ such that $E$ is a stationary set of cofinality $\aleph_{1}$ ordinals, and $\alpha \in E \Longrightarrow$ ot $\left(D_{\alpha}\right)=\beta$ for some fixed $\beta$. Now let $C_{\alpha}=D_{\alpha}$ if ot $\left(D_{\alpha}\right) \leq \beta$, and $C_{\alpha}=D_{\alpha}-(\gamma+1)$ if $\operatorname{ot}\left(D_{\alpha}\right)>\beta$ and $\gamma$ is the $\beta^{\text {th }}$ element of $D_{\alpha}$. Check that $\vec{C}$ is a $\square_{\lambda}(E)$-sequence.

Now fix some $E, \vec{C}$ as in this claim.

Claim 3.3. There exists $\left\langle S_{\alpha}: \alpha \in E\right\rangle$ such that $\vec{S}$ is a $\diamond(E)$-sequence.

Proof. Apply Theorem 32 from [5].

Fix $\vec{S}$ as in this claim. We will construct the tree $T$ along with the functions $x_{\alpha}: \aleph_{1} \longrightarrow T_{\alpha}$ by induction on the levels, maintaining the following hypotheses.

1. The elements of $T$ are the members of $\lambda^{+}$, and $\left|T_{\alpha}\right| \leq \lambda$ for all $\alpha$. The ordinals appearing in $T\left\lceil\alpha\right.$ form an initial segment of $\lambda^{+}$.

2. If $\rho<\sigma$ then there exists $i<\aleph_{1}$ such that $j>i$ implies $x_{\rho}(j)<_{T} x_{\sigma}(j)$.

3. $T \uparrow \alpha$ is normal.

4. For every limit $\alpha$ and every $x \in T \uparrow \alpha$ there exists a "canonical branch" $b^{x, \alpha}=\left\{b_{\gamma}^{x, \alpha}: \gamma \in C_{\alpha}, \gamma \geq \operatorname{ht}_{T}(x)\right\}$ such that

(a) $b^{x, \alpha}$ is cofinal in $T\lceil\alpha$.

(b) $b_{\gamma}^{x, \alpha} \in T_{\gamma}$

(c) $x \leq_{T} b_{\min \left(C_{\alpha}-\mathrm{ht}(x)\right)}^{x, \alpha}$.

(d) For all $\gamma, b_{\gamma}^{x, \alpha}$ is the least element of the level $T_{\gamma}$ which is above $b_{\delta}^{x, \alpha}$ for all $\delta \in[\mathrm{ht}(x), \gamma) \cap C_{\alpha}$.

5. For all $\beta \notin E$, if $\alpha \in \lim \left(C_{\beta}\right)$ then $x_{\alpha}(i)<x_{\beta}(i)$ for all $i<\aleph_{1}$.

A few remarks on the Hypotheses are in order. As usual in this type of construction, we will maintain the truth of Hypothesis 4 by putting a point of $T_{\alpha}$ over each canonical branch of $T\left\lceil\alpha\right.$ whenever $\alpha \notin E$. Since for all $\alpha$ we have $\lim \left(C_{\alpha}\right) \cap E=\emptyset$, the limit stages in the inductive construction of a canonical branch will then be unproblematic, so canonical branches will always exist.

The key point is that Hypothesis 5 is only to apply when $\beta \notin E$. This will be convenient, in that Hypothesis 5 would present difficulties at a stage where we are trying to seal off an antichain. Observe that if $\gamma, \delta \in \lim \left(C_{\alpha}\right)$ and $\gamma<\delta$ then $\delta \notin E$ and $\gamma \in \lim \left(C_{\delta}\right)$, so that if Hypothesis 5 holds we have $x_{\gamma}(i)<x_{\delta}(i)$ for all $i$. 
We describe the inductive construction of $T$ and $\vec{x}$.

1. $\beta=0 . T_{0}$ has a single point and $x_{0}$ is constant with value that point.

2. $\beta=\alpha+1$. Put two points of $T_{\beta}$ over each point of $T_{\alpha}$ and for each $n$ designate one of the successors of $x_{\alpha}(n)$ to be $x_{\beta}(n)$.

3. $\operatorname{cf}(\beta)=\omega, \lim \left(C_{\beta}\right)$ unbounded in $\beta$. Put a point of $T_{\beta}$ over each canonical branch $b^{x, \beta}$, and also over the cofinal branches $\left\langle x_{\gamma}(i): \gamma \in \lim \left(C_{\beta}\right)\right\rangle$ for each $i<\aleph_{1}$. Designate the point which is put over the cofinal branch $\left\langle x_{\gamma}(i): \gamma \in \lim \left(C_{\beta}\right)\right\rangle$ as $x_{\beta}(i)$. As in Case 3 from the construction for Theorem 2.2, it is easy to see that Hypotheses 2 and 5 are satisfied.

4. $\operatorname{cf}(\beta)=\omega, \lim \left(C_{\beta}\right)$ bounded in $\beta$. Put a point of $T_{\beta}$ over each canonical branch $b^{x, \beta}$. Let $\gamma=\max \left(\lim \left(C_{\beta}\right)\right)$, and choose $\left\langle\beta_{n}: n<\omega\right\rangle$ increasing and cofinal in $\beta$ with $\beta_{0}=\gamma$.

Since there are only countably many pairs $\left(\beta_{m}, \beta_{n}\right)$, we may find $i<\aleph_{1}$ so large that if $j>i$ then $x_{\beta_{m}}(j)<_{T} x_{\beta_{n}}(j)$ whenever $m<n$. Now for all $j>i$, put a point over the cofinal branch $\left\langle x_{\beta_{m}}(j): m<\omega\right\rangle$ and designate it as $x_{\beta}(j)$. If $j<i$ then designate the point that was put over $b^{x_{\gamma}(j), \beta}$ as $x_{\beta}(j)$. Hypotheses 2 and 5 are satisfied as in Case 4 from the construction for Theorem 2.2.

5. $\operatorname{cf}(\beta) \geq \aleph_{1}$, and $\beta \notin E$ or $S_{\beta}$ is not a maximal antichain of $T \uparrow \beta$. Put a point on top of each canonical branch, and on top of the branch $\left\langle x_{\gamma}(i): \gamma \in \lim \left(C_{\beta}\right)\right\rangle$ for each $i<\aleph_{1}$. Designate the point that was put over $\left\langle x_{\gamma}(i): \gamma \in \lim \left(C_{\beta}\right)\right\rangle$ as $x_{\beta}(i)$. It is clear that Hypotheses 2 and 5 are satisfied.

6. $\operatorname{cf}(\beta)=\aleph_{1}, \beta \in E, S_{\beta}$ is a maximal antichain of $T \uparrow \beta$. For each $z \in T \uparrow \beta$ choose $w(z) \geq_{T} z$ such that $w(z)$ is also above some point of $S_{\beta}$. Put a point $u(z)$ over the branch $b^{w(z), \beta}$.

Choose $\left\langle\beta_{i}: i<\aleph_{1}\right\rangle$ increasing and cofinal in $\beta$. Given $i<\aleph_{1}$, choose $j(i)$ to be the maximal $j \leq i$ such that $x_{\beta_{0}}(i)<_{T} \ldots<_{T} x_{\beta_{j(i)}}(i)$. Define $x_{\beta}(i)=$ $u\left(x_{\beta_{j(i)}}(i)\right)$. Hypothesis 2 is satisfied as in Case 4 from the construction for Theorem 2.2. Hypothesis 5 is satisfied vacuously because $\beta \in E$.

It is routine to check that we have satisfied the Hypotheses, so in particular $T$ has an $\aleph_{1}$-ascent path. As usual, if $S$ is maximal in $T$ then for some $\beta$ we have that $S \cap \beta=S_{\beta}$ and $S_{\beta}$ is maximal in $T\left\lceil\beta\right.$, so that $\left|S_{\beta}\right| \leq \lambda$. This concludes the proof of Theorem 3.1.

The proof of the following result is very similar (and in fact is exactly parallel to Baumgartner's construction from [2]).

Theorem 3.4. Let $\lambda$ be a cardinal such that

1. $\operatorname{cf}(\lambda)>\omega$.

2. $\gamma<\lambda \Longrightarrow \gamma^{\aleph_{0}}<\lambda$.

3. $2^{\lambda}=\lambda^{+}$.

4. $\square_{\lambda}$ holds.

Then there exists an $\lambda^{+}$-Souslin tree with an $\omega$-ascent path.

\section{REFERENCES}

[1] J. Baumgartner, J. Malitz and W. Reinhardt, Embedding trees in the rationals, Proceedings of the National Academy of Sciences 67 (1970), pp 1748-1753. MR 47:3172

[2] K. J. Devlin, Reduced products of $\aleph_{2}$-trees, Fundamenta Mathematicae 118 (1983), pp 129134. MR 85i:03156 
[3] C. Gray, Iterated forcing from the strategic point of view, $\mathrm{PhD}$ thesis, University of California, Berkeley, 1980.

[4] A. Levy, Basic set theory, Springer-Verlag, Berlin, 1979. MR 80k:04001

[5] S. Shelah, On successors of singular cardinals, in Logic Colloquium '78 (ed: M. Boffa, D. van Dalen and K. McAloon), North-Holland, Amsterdam, pp 357-380. MR 82d:03079

[6] S. Shelah and L. Stanley, Weakly compact cardinals and non-special Aronszajn trees, Proceedings of the American Mathematical Society 104 (1988), pp 887-897. MR 90e:03060

Mathematics Institute, Hebrew University, Givat Ram, 91904 Jerusalem, Israel

E-mail address: cummings@math.huji.ac.il

Current address: Department of Mathematical Sciences, Carnegie Mellon University, Pittsburgh, Pennsylvania 15213-3890

E-mail address: jcumming@andrew.cmu.edu 